Tropical Journal of Pharmaceutical Research May 2021; 20 (5): 1091-1100

ISSN: $1596-5996$ (print); 1596-9827 (electronic)

(C) Pharmacotherapy Group, Faculty of Pharmacy, University of Benin, Benin City, 300001 Nigeria.

Available online at http://www.tjpr.org

Review Article

http://dx.doi.org/10.4314/tjpr.v20i5.30

\title{
Mesoporous silica nanoparticles: Synthesis, functionalization, drug loading and release - A review
}

\author{
Bogdana B Galabova \\ Medical College, Medical University of Sofia, J Filaretova 3,1606, Sofia, Bulgaria
}

*For correspondence: Email: bbgalabova@gmail.com; Tel: +359-889665844

Sent for review: 2 January 2021

Revised accepted: 26 April 2021

\begin{abstract}
A brief survey on the synthesis of several types of mesoporous silica nanoparticles and their uses as drug delivery nanocarriers is presented in this study. Mesoporous silica nanoparticles (MSNs) have a small pore size, resulting in a large surface area and porosity. These characteristics are easily adjustable, conferring on MSNs advantages over other nanoparticles for use in medicine and pharmacy. Previous and latest trends in the technology of this type of nanoparticles, and the possibilities for their application in drug loading are considered, as well as methods for increasing drug loading capacity, functionalization and release of bioactive substances. It has been found that the method used for the synthesis of some types of MSNs results in better drug loading. The functionalization of nanoparticles not only allows for more successful loading of bioactive substances in its pores, it also successfully controls drug release. Moreover, these versatile nanoparticles enhance the solubility of poorly-soluble drugs, thereby overcoming one of the biggest problems in the absorption of these drugs.
\end{abstract}

Keywords: MCM-41, HMS, SBA, Synthesis, Soft templating, Hard templating

\begin{abstract}
This is an Open Access article that uses a funding model which does not charge readers or their institutions for access and distributed under the terms of the Creative Commons Attribution License (http://creativecommons.org/licenses/by/4.0) and the Budapest Open Access Initiative (http://www.budapestopenaccessinitiative.org/read), which permit unrestricted use, distribution, and
\end{abstract} reproduction in any medium, provided the original work is properly credited.

Tropical Journal of Pharmaceutical Research is indexed by Science Citation Index (SciSearch), Scopus, International Pharmaceutical Abstract, Chemical Abstracts, Embase, Index Copernicus, EBSCO, African Index Medicus, JournalSeek, Journal Citation Reports/Science Edition, Directory of Open Access Journals (DOAJ), African Journal Online, Bioline International, Open-J-Gate and Pharmacy Abstracts

\section{INTRODUCTION}

In recent years, mesoporous silica nanoparticles (MSNs) have been extensively studied as nanocarriers for delivering bioactive substances due to their attractive physicochemical properties $[1,2]$. The MSNs are mechanically, thermally and chemically stable particles. They have large surface areas, extensive pore volumes and narrow distribution of adjustable pore diameters, thanks to their specific structure [1]. The MSNs can be used to develop systems for controlled drug delivery which ensures the sustained release of drugs by maintaining their therapeutic concentrations at the target areas for an extended period, leading to reduction in frequencies of doses and the possibility of side effects $[3,4]$. These systems can also solve the problem of poor aqueous solubility of drugs by loading them into the mesoporous silica nanoparticle pores. Limited internal space improves drug dissolution, probably due to a decrease in its degree of crystallinity $[5,6]$.

This review was aimed at providing methods for synthesis of various types of MSNs used as drug delivery systems, loading of bioactive substances, functional group grafting, and drug release. 


\section{SYNTHESIS OF MSN}

Stober is the pioneer in the synthesis of silica particles with uniform microsize and spherical shapes. Thus, this method of synthesis is attributed to him. It is based on the hydrolysis of tetraalkyl silicates in a hydroethanolic medium, and use of ammonia as a catalyst. Based on this approach, many modifications have been constantly made. The first of the many modifications was made by Grun et al, who added $\mathrm{n}$-hexadecyltrimethylammonium bromide and $n$-hexadecylpyridinium chloride as cationic surfactants to the reaction mixture as templates, resulting in MCM-41 structure [7].

Thereafter, MSNs with different structures have been developed using different types of surfactants. The design of MSNs, their morphologies, and pore sizes and structures, vary because of various designs and various controls in the synthesis process (Figure 1).
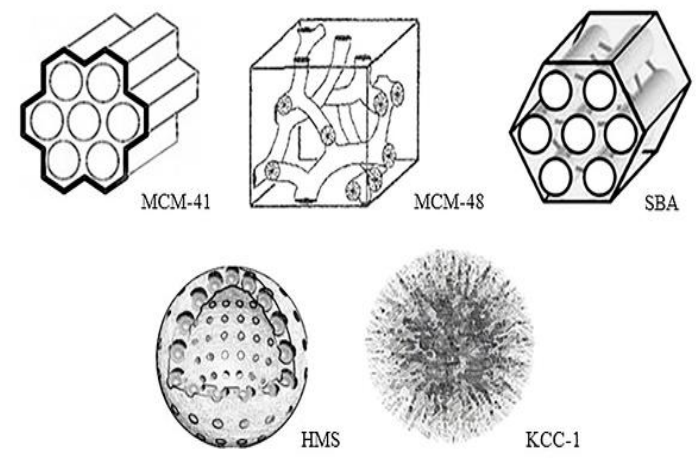

Figure 1: Types of MSNs

The MCM-41 is the most studied type of MSNs. They have an ordered arrangement of uniform two-dimensional (2D) hexagonal mesopores. Firstly, they are prepared using cetyltrimethylammonium bromide (CTAB), tetraethyl orthosilicate (TEOS) and a catalyst. Sodium metasilicate $\left(\mathrm{Na}_{2} \mathrm{SiO}_{3}\right)$ is used ase silica precursor as well [8]. The team of Kresge used a solution containing hexadecyltrimethylammonium ion $\mathrm{C}_{16} \mathrm{H}_{33}\left(\mathrm{CH}_{3}\right)_{3} \mathrm{~N}^{+} \mathrm{OH} / \mathrm{Cl}$ mixed with catapal alumina tetramethylammonium silicate solution, and precipitated silica at 1:1 molar ratio of tetramethylammonium: $\mathrm{SiO}_{2}$. The combination was heated at a temperature of $150^{\circ} \mathrm{C}$ for $48 \mathrm{~h}$ under pressure in a sterilizer. After cooling to room temperature, filtering, washing with water and air-drying, the solid product was calcinated at $540{ }^{\circ} \mathrm{C}$ for $1 \mathrm{~h}$, first in flowing nitrogen, and thereafter in flowing air [9]. It was found that substituting dodecyl trimethylammonium with hexadecyltrimethylammonium ion led to changes in pore diameter of MCM-41, and it was concluded that varying the length of the alkyl chain of cationic type surfactants allows for nanoparticles with different pore sizes. Another way of increasing the diameter of the pores is by adding $1,3,5$-trimethylbenzene. It was suggested that organic molecules should be solubilised in the hydrophobic inner part of micelles, causing an increased micellar size [9].

It is known that CTAB self-aggregates into micelles when its concentration reaches the critical level for micelle formation (Figure 2). The silica precursors superpose at the surface of surfactant around the micellar polar head and form a wall around them. Removal of the surfactant results in formation of MCM- 41 .

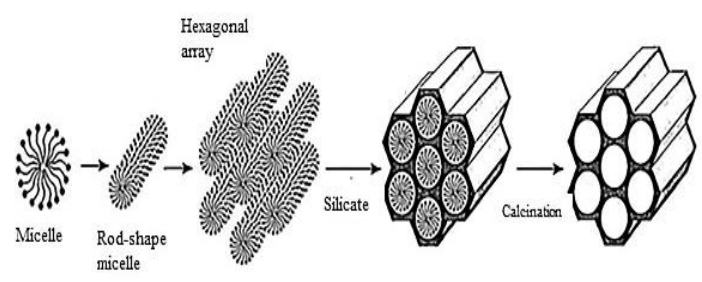

Figure 2: Scheme for synthesis of MCM-41 with a template of CTAB micelles

By tailoring the surfactant/silica molar ratio, the diameter of MCM-41 and morphology can be controlled, as well as the co-condensation of tetraethoxysilane and hydrophobic organoalkoxysilane. Other opportunities for precise control of the size, shape of the inner structure, pore size and geometry include the use of organic swelling agents or addition of cosolvents, $\mathrm{pH}$ control, and regulation of temperature, as well as drying and stirring rates. Larger pores and higher specific surface areas are produced when $\mathrm{Na}_{2} \mathrm{SiO}_{3}$ is used as a precursor due to increase in the aggregate number of the surfactant micelles, whereas the use of TEOS results in lower surface areas and smaller pore sizes [10]. By adjusting the concentration and the molar ratio of CTAB and $\mathrm{NaOH}, \quad$ MCM-41with spherical-to-rod-like structures can be generated [11].

The HMS is a type of MSNs with a hollow interior and mesoporous shell. The main distinguishing characteristics of HMS are low density, high specific area and the possibility of high loading capacity [2, 3]. Methods for preparing HMS include soft templating, hard templating, selftemplate method, layer-by-layer method [12]; Kirkendall effect [13], Ostwald ripening [14], and galvanic replacement [15]. 


\section{Soft-template method}

Dual or multi- surfactants are used to form a complex template and to build a shell with mesopores and hollow inner parts (Figure 3 ).

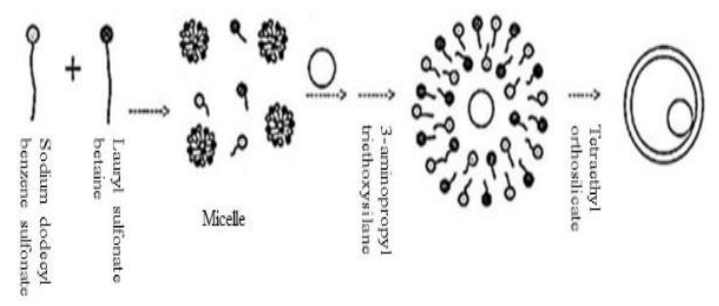

Figure 3: Synthesis of HMS using soft template method

During the first stage, $\mathrm{SiO}_{2}$ is dispersed in an aqueous mixture of laurylsulfonate betaine and sodium dodecylbenzene sulfonate at a molar ratio of 1:1. In the second stage, 3aminopropyltriethoxysilane (APS) is added to induce formation of movable core vesicles. By electrostatic interaction, APS adheres to the vesicle surface. Sol-gel transition after hydrolysis of APS and TEOS produce step $\mathrm{SiO}_{2}$ shells in the last. After removal of surfactants, HMS is formed [16].

\section{Single micelle templating}

Yang and co-workers used pluronic copolymer at various EO:PO ratios and organosilica precursor [17]. No binding took place between the micelles and the precursor of silicate. A positively charged surfactant kept the micelle/silica isolated [18]. Thus, hollow silica nanoparticles were obtained after pyrolysis. The method of single micelle templating forms tiny hollow mesoporous silica nanoparticles because of the size of micelles. The HMS nanoparticles produced by this method generally have diameters under $20 \mathrm{~nm}$.

\section{Vesicle-templating}

It is possible to increase the size of the resulting particles by forming vesicles. The addition of anionic surfactant lowers the curvation of mesostructure templates. Hollow mesoporous spheres with porous shells are capable of direct incorporation of amino groups. This functionalization of silica occurs after anionic surfactant extraction with acids [19]. Uniform HMS is formed after co-condensation of TEOS and organotriethoxysilanes. The synthesis results in a solution of triethanolamine and cationic surfactant cetyltrimethylammonium chloride.

\section{Microemulsion-templating}

When the nanoparticles have to be less than 100 $\mathrm{nm}$ in size, the microemulsion method is preferred. Water-in-oil-water emulsion can be used to synthesize porous silica nanomaterials with a hollow inner structure. It is difficult to remove the soft template entirely. Achieving this is often associated with reduction in dispersion. The material obtained through the soft templating method yields an amorphous or semicrystalline phase because of the narrow temperature range used during synthesis. High-temperature calcination is used to eliminate the amorphous phase, but the mesoporous structure is not stable at these extreme conditions. To improve the stability of the particles obtained, the socalled hard template method has been developed.

\section{Hard templating}

Various polymer networks, silicate colloids, and metal oxides are used as solid templates. To obtain homogeneous particles, the silicification on the surface of the organic template must proceed at a higher rate than that of silica selfassociation. Besides, organic templates must be stable during the process of silica accumulation and condensation. Due to the frequent interactions between the silicate precursor and the organic template, the organic components are extracted from the solution. The formation of a structure becomes impossible, and the synthesis fails. The separation of the template must be feasible after the silica condensation phase. Most often, this can be achieved in one of the following ways: acid dissolution, calcination at high temperature or extraction. The free volume and the free surface of the obtained particles depend on the correct course of this stage.

\section{Polymer bead templating}

This successfully results in small and uniform particles with the help of polystyrene and polymethacrylate using the method of rigid template, since it is easily removed after calcination at $500^{\circ} \mathrm{C}$. The template calcination method must result in a stable structure, clean pores and a large number of silanol groups which are essential for preparing systems for biomedical applications. Calcination at a high temperature of $500^{\circ} \mathrm{C}$ is the most commonly used, but very slow method for template removal [20]. Rapid calcination has been studied as a variant of the method in which samples are subjected to short-term intensive calcination with rapid heating [21]. Another variant of this method 
is microwave-assisted calcination, in which the organic template is removed in a few minutes, but with the use of special equipment [22].

\section{Metal and metal oxide templating}

The surface activation of solid templates can be extended to encapsulate metal particles, metal oxide particles or semiconductor nanoparticles in the HMS structure. Thus, the advantages of reservoir mesoporous silicates and other functional nanoparticles are combined to obtain a nanocomposite with specific properties. The synthesis of these nanoparticles involves the use of various surfactants and polymers as agents between the metal nanoparticles on which the mesoporous structure of the reservoir wall is built. Thus, the metal functional particles remain embedded in the reservoir structure. The complex particles obtained in this way have significant advantages over individual nanoparticles: the silicate wall has increased thermal stability, and prevents direct contact between metal nanoparticles and organic material, thereby increasing biocompatibility, free surface and the possibility of surface modification. In the procedure, it is possible to obtain HMS using acid extraction of the nanoparticles located in the reservoir. Unlike calcification, the method of extraction with organic solvent can also be used with prefunctionalised materials [23]. This is due to the fact that it is possible to remove the organic template without decomposition of the cocondensed organic part. The disadvantages of this method are incomplete removal of the template, even with repeated extraction, as well as the long duration of the process. Quaternary ammonium surfactants are usually used as coating agents. Kim's research team has proposed a simple method for preparation of monodisperse $\mathrm{Fe}_{3} \mathrm{O}_{4}$ silicate nanoparticles using cetyltrimethylammonium bromide as a stabiliser, along with a structuring agent [24]. In addition to complex silicate particles containing iron nanostructures, those based on silver, gold and platinum nanoparticles have also been developed.

Wang et al developed the preparation of reservoir silica particles using gold nanoparticles and cetyltrimethylammonium bromide as templates for the core, and mesopores in the silica wall [25]. Gold particles are appropriate for obtaining hollow systems because of the possibilities they afford for controlling the largeness of the particles, as well as good size distribution, spherical shape, and high melting temperature. The synthesis is followed by a calcination step to separate the organic components and empty the mesopores. The particles are then etched to separate the metal component and drain the core.

\section{Layer-by-layer method}

Layer-by-layer-based hollow mesoporous silica nanoparticles consist of multilayer shells and cavities. The exterior is strengthened through consecutive adsorption of contrariwise charged components around a negatively loaded mesoporous silica sphere template. They are held together due to the strong electrostatic forces created between the component layers [26]. The cavity obtained after removing the mesoporous silica sphere template represents the main volume of the capsules where bioactive substances can be encapsulated.

Javier et al subjected mesoporous silica spheres to catalase solution, resulting in immobilisation of the enzyme in them. The catalase-loaded mesoporous particles are coated with three poly (L-lysine)/poly (L-glutamic acid) layers. Thereafter, the porous core is removed by exposure to a hydrofluoric acid/ammonium fluoride buffer, pH 5 [27]. This results in HMS. Thanks to the high versatility of the layer-by-layer technique, the two parts can easily be adapted to meet different specific application requirements.

\section{Self-template method}

The self-template method is a procedure used for the synthesis of hollow silica nanoparticles without a different template. Mesoporous silica particles coated with cationic polydimethyldiallylammonium chloride in an alkaline medium can transfer the nanoparticles to cavity structure. Hydroxyl ions dissolve silica oligomers. They are negatively charged, and so they sediment on the positively charged layer, forming crosslinked silica shell. [28]. For selectively etching, $\mathrm{NaOH}$ is used, and a hollow structure is formed under PVP protection. These strategies are called surface-protected etching [29]. Yu et al developed preservers using acidic and hydrothermal treatment to make a selective etching of the inner space, and transformed silica nanoparticles to hollow structures. [30]. Wong et al transformed hollow structures from solid silica for 30 min after establishing that the inner layer was pulpous with a sponge structure and that it was possible to selectively etch it with hot water. The outer layer was most rigid due to the formation of silicic acid aggregates after the Stober reaction. Thus, it was demonstrated that Stöber silica particle is not homogeneous, and that it is possible to produce hollow nanostructures from them using the self-template 
method [31]. The advantages of the self-template methods are their cost and simplicity.

\section{Kirkendall effect}

Kirkendall effect is a diffusion phenomenon that explains the interaction among atoms in two contiguous phases with different diffusivities. When the atoms in an inner layer diffuse quickly into an outer layer, they move slowly to the inner layer. During the diffusion process, the supersaturation of lattice vacancies develops into an interior pore which becomes the inner part of the final hollow structure [32]. The selforganisation method and the promoter Kirkendall effect have been reported for the first time, to form hollow nanostructures Yin et al [33]. Yoonkook Son et al have demonstrated the use of the same effect for silica and germanium nanoparticles via the introduction of nanoscale surface layers of $\mathrm{SiO}_{2}$ and $\mathrm{GeO}_{2}$, respectively. The $\mathrm{Si}$ and $\mathrm{Ge}$ atoms diffuse across the oxide layers and form pores in the cores. The Kirkendall effect allows nanoparticles to be transformed into hollow ones.

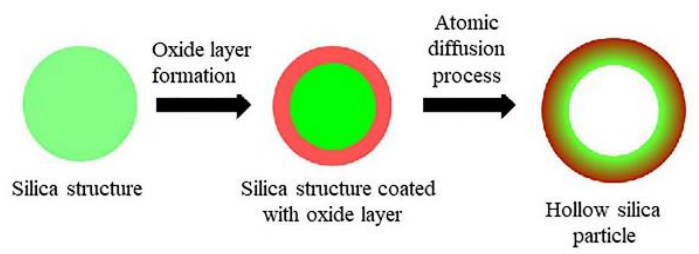

Figure 4: Transformation into hollow silica nanoparticles through the Kirkendall effect

\section{Ostwald ripening}

Ostwald ripening is a well-known phenomenon that explains the conversion of a non-uniform structure into a mixture of two crystalline solids that co-exist as a new crystalline solid or liquid colloids, and it involves matter relocation. The IUPAC in 2007 recommended the definition of Ostwald ripening as the "dissolution of small crystals or sol particles and the re-deposition of the dissolved species on the surfaces of larger crystals or sol particles" [34]. The ripening process is important since macroscale particles are more energetically privileged than microscale particles and cause increasing solubility for the latter. During different experimental conditions for sol-forming particles in a solution, many reversible chemical reactions take place on the solid/liquid boundary. Due to the diverse range of crystallites, there are changes in amount of solutes. The uniformisation of these concentration gradients will lead to total dissolution of smaller crystallites due to the growth of the large crystallites. The possibility of displacement of matter is used as an opportunity to form cavities, where the crystals are low dimensional or closely packed if Ostwald ripening continues [35].

Hollow nano-structured materials obtained after Ostwald ripening are already of interest to research teams. Lou et al have carried out a reasonable production of hollow silicon spheres. They coated gold and platinum nano-sized particles with a silica exterior, and subsequently, two, three or four of the particles were connected through self-association. The same team investigated Ostwald ripening in a closed microspace using chloride as a binding ligand in gold hollow silica particles with many cores. Moreover, they used as-prepared multicore Auhollow silica particles to study Ostwald ripening in confined micro-space, in which chloride is an efficient mediating ligand [36].

Gold nanoparticles grown on PS nanospheres are used as removable templates for silica coating via a modified Stöber method. Uniform gold nanoparticles are grown on the surface of amino-functionalised monodisperse PS nanospheres. The resultant model multicore hollow spheres are obtained with hightemperature removal of the PS-Au-silica core/shell nanoparticles at $450{ }^{\circ} \mathrm{C}$ for two hours [37]. Following Ostwald ripening, a significant fraction of Au nanoparticles assumes a triangular or rod-like shape.

\section{Galvanic replacement}

The galvanic replacement reaction can be used for the synthesis of hollow nanoparticles too. The Xia research group has created various hollow nanoparticles of silver, gold, platinum, and paladium using galvanic substitution reaction. They managed to control the pore volume and the size of the particle walls [38].

The team of Hyeon worked with nanosized silica as a template for obtaining hollow nanoparticles. Their method, after a thermal regime, allows silica layer elimination and variation in structure and morphology of the resultant particles. [39]. They used $\beta-\mathrm{FeOOH}$ nanoparticles with a silica coat. After five hours at $300^{\circ} \mathrm{C}$, hollow particles were obtained because of the de-hydroxylation of $\beta-\mathrm{FeOOH}$. Received nanocapsules were processed at $500^{\circ} \mathrm{C}$ and resulted in magnetite $\left(\mathrm{Fe}_{3} \mathrm{O}_{4}\right)$ formation. The silica coat was separated after sonication of coated particles in $\mathrm{NaOH}$ solution. Similarly, Yang et al obtained hollow silica particles in the nanosize range [40] using $\mathrm{Fe}_{3} \mathrm{O}_{4}$ nanoparticles as an inner template. 
Stöber method was used for the synthesis of bonded iron particles together with polyvinylpyrrolidone coated with silica. A large cavity was obtained after removal of iron core with $\mathrm{HCL}$, and high-temperature calcination of the silica shell.

\section{SURFACE FUNCTIONALISATION, DRUG LOADING AND DRUG RELEASE}

The MSNs comprise an open system of pores that allows the loading of drugs. These drugs can quickly diffuse, depending on the solvent and the type of drug $[2,3,5,6]$. One of the advantages of mesoporous silica nanoparticles is their high loading capacity, based on the significant pore volume. Their dilatation results in higher loading and retention of bioactive substances. The expansion of pores in silica material can be achieved with agents such as ethanol, alkanes, triisopropylbenzene, trioctylamine and dimethylhexadecylamine [41].

Obtaining HMS itself is one strategy used for increasing load capacity. The hollow cavities in HMS provide significantly more space for loading of bioactive substances than MSN. Water-soluble and water-insoluble bioactive substances can be successfully loaded into the pores or cavities of these particles, based on their adsorption properties.

After removal of the solvent, the particles immersed in the bioactive substance solution remain impregnated with it. This simple method has continued to be the most popular way to load drugs into porous silica particles $[2,3,5]$. The use of a non-solvent most often allows the loading of the drug substance [6]. In parallel with the preparation of mesoporous silica particles, the bioactive substance could be successfully loaded. This combined method has the advantage of excluding the use of organic solvents. However, it still depends on the interaction between silanol or other groups of silica walls and functional groups in the bioactive substance, and the type of surfactant used.

As a variant of this method is known the loading after melting of the substance after microwave heating [42]. It is also found that increasing the amount of bioactive substance relative to mesoporous particles significantly improves the success rate of loading [43]. By creating an opportunity for interaction between the functional groups of the bioactive substance and those on the surface of the particles, the loading, and especially the retention of the drug in the pores is significantly improved. Surface functionalisation of MSNs with functional groups like 3- aminopropyl, 2-cyanoethyl, 3 -cloropropyl, 3merkaptopropyl, alkyl, phenyl, vinyl and allyl enhances the loading and release of drugs.

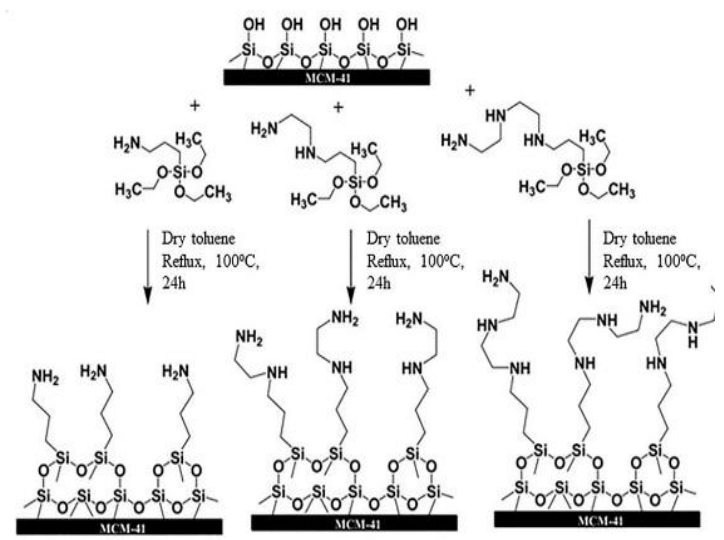

Figure 5: Amino-functionalization of the surface of MCM-41

The functional groups grafted on the silica surface interact with the drug molecules through ionic, electrostatic connections, or esterification, and in this manner, they effectively control the drug release or drug retention into pores of MSNs during loading process [44]. The loading of a bioactive substance, followed by functionalization results in enhanced successful loading, when compared to a load of already grafted particles [45]. Results have been published indicating a $10 \%$ improvement in 5-FU loading in amino-functionalised MSNs, due to the electrostatic connections between the negatively charged drug and the positively charged carrier. Oppositely charged interactions are possible between different types of functional groups grafted on the surface of the particles, and those present in the chemical formula of drugs [46].

Other research teams used carboxy modification of mesoporous silica to show how the adsorption of famotidine, a drug molecule with an amino group, depends on the degree of functionalization. The acid-catalysed hydrolysis of cyano carriers effects their carboxylation to carboxylic acid groups [47]. Carboxylations of MSM-41 and SBA-15 were achieved after the use of succinic anhydride and a ring-opening reaction. A bioactive substance with an amino group was selected for loading, expecting an electrostatic interaction between the functional groups of the carrier and the drug. This modification allowed for improved loading of sulfadiazine in MSM-41, but did not change the degree of SBA loading. In vitro release studies showed that functionalisation of MCM-41 and SBA-15 silica with carboxylic groups makes them 
appropriate carriers for extended drug release [48].

If the surface of the MSN is initially functionalised, then the bioactive substance is absorbed, with possibilities that the drug will get on the surface of MSNs. The burst effect will occur in this case and will compromise prolonged release. On the other hand, drug loading, followed by surface functionalisation, maintains slow and sustained drug release. Loading the drug into the pores and closing the pores with APTES stops the drug from being released. Variations in amount of APTES are essential in management of drug release from the pores [49].

Ibuprofen ordinary is incorporated in nanoparticles in the hope that its $\mathrm{COOH}$ links up with the $\mathrm{Si}-\mathrm{OH}$ groups of MSNs. Often ibuprofen - ibuprofen interactions form dimers by virtue of hydrogen bonds of their $-\mathrm{COOH}$ groups [94]. A research team found that dimers were not linked to the wall of MSNs, but physiosorbed on the surface. These systems showed expressed burst effect. This problem can be resolved with the amino functionalization of MCM-41. Carboxy groups of Ibuprofen are linked to the amino groups of functionalized MCM-41 by ionic interaction between them. Effective release control is due to stronger electrostatic interactions than the hydrogen bonds between the dimers of Ibuprofen.

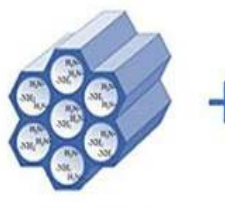

$\mathrm{MCM}-41-\mathrm{NH} 2$

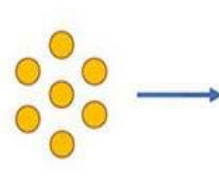

Ibuprofen

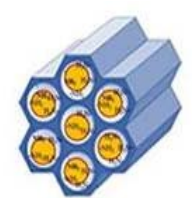

$\mathrm{MCM}-41-\mathrm{NH} 2 \mathrm{Ibu}$
Figure 6: Loading of ibuprofen into aminofunctionalized MCM-41

During the synthesis, amino functionalised SBA15 showed a release of the bioactive substance within $10 \mathrm{~h}$, while SBA-15 modified after the synthesis released ibuprofen for three days [51]. The use of hydrophobic substances can successfully manage drug liberation. This type of functionalization inhibits water penetration into the pores, thereby blocking easy discharge of the drug. Based on this strategy, SBA-15 is functionalised with octyl and octadecyl moieties to effectively control the release rate of erythromycin. On the other hand, long-chain hydrocarbon moieties (C8 and C18) decrease the pore size of silica particles [52].

The drug must diffuse from the pores to be released from the carrier. If a polymer is added after loading to retain the bioactive substance in the pores of the silicate material, this would improve the loading capacity and allow a delayed release. The polymer can interact with the drug or with the silica surface. The MSNs can be tailored through polymer modification for controlling drug release by post-coating of mesoporous particles preliminarily loaded with a drug. Surface modification of the mesoporous nanoparticles with carbopol is used to provide a low initial release and longer budesonide residence in the gastrointestinal tract. Using polyacrylic acid, MSNs can be tailored to control drug release from post-coated mesoporous particles preliminarily loaded with a drug. Carbopol is used effectively to reduce burst release and retain budesonide in the intestines for an extended period, while guaranteeing prolonged absorption. Swelling of polymer results in the formation layers around nanoparticles, and even if there is an immediate release of the bioactive substance, the layer is retained around the carrier. The concentration of this coated system was increased in inflamed colon tissue, as revealed after histological analysis [53].

Carbopol coating of MCM-41 loaded with budesonide has been successfully made by the team of Yoncheva. The polymer layer formed around nanoparticles have free $\mathrm{COOH}$ groups and form bonds with $\mathrm{NH}_{2}$-functionalized silica carrier. Functionalization is a highly successful approach for sustaining the release of budesonide and subsequent polymer coating of mesoporous silica carriers [54].

The newly developed drug-delivery systems for pramipexole based on HMS have been additionally subjected to coating with two bioadhesive polymers to achieve sustained release. The double-layer reduced the initial burst effect and successively lowered pramipexole release [3]. Chitosan and sodium alginate electrostatically react and form an interpolymer complex that prevents quick liberation of the bioactive substance.

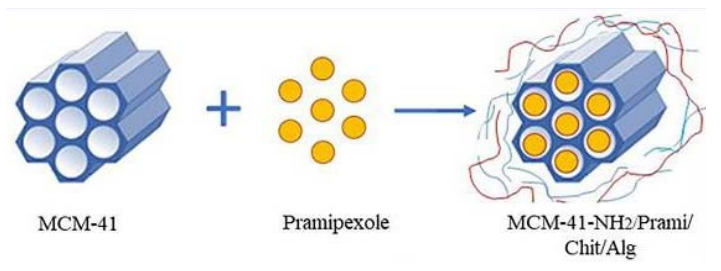

Figure 7: Double-coated MCM-41 loaded with pramipexole

Interpolymer complex formation between PAA or PMA and AA results in hydrogel formation around nanoparticles sensitive to $\mathrm{pH}$. In principle, 
it can be used as a polymeric coating for different types of MSNs [3,55-57].

\section{CONCLUDING REMARKS}

This review examines the synthesis and functionalization of the most-studied mesoporous silica particles as drug delivery systems. Functionalization makes the particles efficient and sensitive systems for site-specific delivery through via various stimuli such as $\mathrm{pH}$, temperature, light and enzymes, and also allows for more effective control of the drug release kinetics. The use of newly synthesised specific polymers can be proposed as a future direction in the development of drug delivery. The new polymers used for the coating of mesoporous particles could offer targeted drug delivery in a hybrid drug delivery system. A combination of inorganic and polymeric materials in the same system could meet specific requirements for the delivery of bioactive substances, thereby combining the advantages of both materials.

\section{DECLARATIONS}

\section{Conflict of interest}

No conflict of interest is associated with this work.

\section{Contribution of authors}

I declare that this work was done by the authors named in this article and all liabilities pertaining to claims relating to the content of this article will be borne by the author.

\section{Open Access}

This is an Open Access article that uses a funding model which does not charge readers or their institutions for access and distributed under the terms of the Creative Commons Attribution License (http://creativecommons.org/licenses/by/ 4.0) and the Budapest Open Access Initiative (http://www.budapestopenaccessinitiative.org/rea d), which permit unrestricted use, distribution, and reproduction in any medium, provided the original work is properly credited.

\section{REFERENCES}

1. Vallet-Regí $M$, Rámila $A, D e l$ Real RP, Pérez-Pariente J. A new property of MCM-41: drug delivery system. Chem Mater 2001; 13(2): 308-311.

2. Voycheva C, Tzankov B, Tzankova D, Avramova K, Yoncheva $K$. Formulation of Tablets Containing
Glimepiride-loaded Mesoporous Silica Particles. Indian J Pharm Sci 2019; 81(3): 483-488.

3. Tzankov B, Voycheva C, Yordanov Y, Aluani D, Spassova I, Kovacheva D, Lambov N, Tzankova V. Development and in vitro safety evaluation of pramipexole-loaded hollow mesoporous silica (HMS) particles. Biotech Biotech Equip 2019; 33(1): 12041215.

4. Popova T, Tzankov B, Voycheva Ch, Spassova IV Kovacheva D, Tzankov S, Denitsa Aluani D, Tzankova $V$, Lambov N. Mesoporous silica MCM-41 and HMS as advanced drug delivery carriers for bicalutamide, $2021 \mathrm{~J}$ Drug Deliv Sci and Technology 62:102340

5. Tzankov B, Voycheva $C$, Aluani D, Yordanov $Y$, Avramova K, Tzankova V, Spassova I, Kovacheva D, Yoncheva K. Improvement of dissolution of poorly soluble glimepiride by loading on two types of mesoporous silica carriers. J Solid State Chem 2019; (271): 253-259.

6. Popova T, Tzankov B, Voycheva C, Yoncheva $K$, Lambov N. Development of advanced drug delivery systems with bicalutamide based on mesoporous silica particles. C R Acad Bulg Sci 2019; 72(12): 1654-1660.

7. Grun M, Lauer I, Unger K. The synthesis of micrometreand submicrometer-size spheres of ordered mesoporous oxide MCM-41. Adv Mater 1997; (9): 254 256.

8. Yanagisawa $T$, Shimizu $T$, Kuroda K, Kato $C$. The Preparation of Alkyltriinethylaininonium-Kaneinite Complexes and Their Conversion to Microporous Materials. Bull Chem Soc 1990; (63): 988.

9. Kresge C, Leonowicz $M$, Roth $W$, Vartuli J, Beck J. Ordered mesoporous molecular sieves synthesised by a liquid-crystal template mechanism. Nature 1992; (359): 710-712.

10. Pang X, Tang F. Morphological control of mesoporous materials using inexpensive silica sources. Micropor Mesopor Mat 2005; (85): 1-6.

11. Huang X, Li L, Liu T, Hao N, Liu H, Chen D, Tang F. The shape effect of mesoporous silica nanoparticles on biodistribution, clearance, and biocompatibility in vivo. ACS Nano 2011; (5): 5390-5399.

12. Javier $A$, Kreft $O$, Semmling $M$, Kempter $S$, Skirtach $A$, Bruns $O$, del Pino $P$, Bedard M, Raedler J, Käs J et al. Uptake of Colloidal Polyelectrolyte-Coated Particles and Polyelectrolyte Multilayer Capsules by Living Cells. Adv Mater 2008; (20): 4281-4287.

13. Yin $Y$, Rioux R, Erdonmez $C$, Hughes $S$, Somorjai G, Alivisatos A. Formation of Hollow Nanocrystals through the Nanoscale Kirkendall Effect. Science 2004; 304(5671): 711-714.

14. Lou X, Yuan C, Rhoades E, Zhang Q, Archer L. Encapsulation and Ostwald Ripening of $\mathrm{Au}$ and $\mathrm{Au}-\mathrm{Cl}$ Complex Nanostructures in Silica Shells. Adv Funct Mater 2006; 16(13): 1679-1684.

15. Chen J, McLellan J, Siekkinen A, Xiong Y, Li Z, Xia Y. Facile synthesis of gold-silver nanocages with 
controllable pores on the surface. J Am Chem Soc 2006; 128(46): 14776-14777.

16. Wu X, Xu D. Formation of Yolk/SiO2 Shell Structures Using Surfactant Mixtures as Template. J Am Chem Soc 2009; (131): 2774-2775.

17. Liu X, Li X, Guan Z, Liu J, Zhao J, Yang Y, Yang Q. Organosilica nanotubes: large-scale synthesis and encapsulation of metal nanoparticles. Chem Commun 2011; 47(28): 8073-8075.

18. Yuan J, Mykhaylyk O, Ryan A, Armes S. Cross-Linking of Cationic Block Copolymer Micelles by Silica Deposition. J Am Chem Soc 2007; 129(6): 1717-1723.

19. Han L, Gao C, Wu X, Chen Q, Shu P, Ding Z, Che S. Anionic surfactants templating route for synthesizing silica hollow spheres with different shell porosity. Solid State Sci 2011; 13(4): 721-728.

20. Chen $Y$, Meng Q, Wu M, Wang S, Xu P, Chen H, Li Y, Zhang L, Wang L, Shi J. Hollow Mesoporous Organosilica Nanoparticles: A Generic Intelligent Framework-Hybridization Approach for Biomedicine. J Am Chem Soc 2014; 136(46) 16326-16334.

21. Bagshaw S, Bruce I. Rapid calcination of high-quality mesostructured MCM-41, MSU-X, and SBA-15 silicate materials: A step towards continuous processing? Micropor Mesopor Mat 2008; (109): 199-209.

22. Gallis K, Landry C. Rapid Calcination of Nanostructured Silicate Composites by Microwave Irradiation. Adv Mater 2001; 13(1): 23-26.

23. Zienkiewicz-Strzałka M, Pikus S, Olszewska E, Barczak M. Mesoporous Ordered Organosilicas Containing Zr and Ti Species. Solid State Phenom 2010; (163): 55-58.

24. Kim J, Kim H, Lee N, Kim T, Kim H, Yu T, Song Ch, Moon W, Hyeon T. Multifunctional uniform nanoparticles composed of a magnetite nanocrystal core and a mesoporous silica shell for magnetic resonance and fluorescence imaging and drug delivery. Angew Chem Int Ed 2008; 47(44): 8438-8441.

25. Wang X, Tan L, Li X, Song N, Li Z, Hu J, Cheng Y, Wang $Y$, Yang $Y$. Smart mesoporous silica nanoparticles gated by pillararene-modified gold nanoparticles for ondemand cargo release. Chem Commun 2016; 52(95): 13775-13778.

26. Donath E, Sukhorukov G, Caruso F, Davis S, Mohwald H. Novel Hollow Polymer Shells by Colloid-Templated Assembly of Polyelectrolytes. Angew Chem Int Ed 1998; (37): 2201-2205.

27. Javier A, Kreft $O$, Semmling $M$, Kempter $S$, Skirtach $A$, Bruns O, del Pino P, Bedard M, Raedler J, Käs J, Plank $C$ et al. Uptake of Colloidal Polyelectrolyte-Coated Particles and Polyelectrolyte Multilayer Capsules by Living Cells. Adv Mater 2008; (20): 4281-4287.

28. Zhang $T$, Ge J, Hu Y, Zhang Q, Aloni S, Yin Y. Formation of hollow silica colloids through a spontaneous dissolution-regrowth process. Angew Chem Int Ed 2008; 47(31): 5806-5811.

29. Zhang Q, Zhang T, Ge J, Yin Y. Permeable Silica Shell through Surface-Protected Etching. Nano Lett 2008; 8(9): 2867-2871.
30. Yu Q, Wang $P$, Hu S, Hui J, Zhuang J, Wang $X$. Hydrothermal Synthesis of Hollow Silica Spheres under Acidic Conditions. Langmuir 2011; 27(11): 7185-7191.

31. Wong Y, Zhu L, Teo W, Tan Y, Yang Y, Wang C, Chen H. Revisiting the Stöber Method: Inhomogeneity in Silica Shells. J Am Chem Soc 2011; 133(30): 11422-11425.

32. Fan H, Gosele U, Zacharias M. Formation of Nanotubes and Hollow Nanoparticles Based on Kirkendall and Diffusion Processes: A Review. Small 2007; 3(10): 1660-1671.

33. Yin Y, Rioux R, Erdonmez C, Hughes S, Somorjai $G$ Alivisatos A. Formation of Hollow Nanocrystals Through the Nanoscale Kirkendall Effect. Science 2004; (304): 711-714.

34. Aleman J, Chadwick A, He J, Hess M, Horie K, Jones R, Kratochvil P, Meisel I, Mita I, Moad G et all. Definitions of terms relating to the structure and processing of sols, gels, networks, and inorganic-organic hybrid materials (IUPAC Recommendations, 2007), Pure Appl Chem 2007; 79(10): 1801-1827.

35. Liu B, Zeng H. Symmetric and Asymmetric Ostwald Ripening in the Fabrication of Homogeneous Core-Shell Semiconductors. Small, 2005; 1(5): 566-571.

36. Lou X, Yuan C, Rhoades E, Zhang Q, Archer L. Encapsulation and Ostwald Ripening of $\mathrm{Au}$ and $\mathrm{Au}-\mathrm{Cl}$ Complex Nanostructures in Silica Shells. Adv Funct Mater 2006; 16, (13): 1679-1684.

37. Stober W, Fink A, Bohn E. Controlled growth of monodisperse silica spheres in the micron size range. $J$ Colloid Interf Sci 1968; 26(1): 62-69.

38. Chen J, McLellan J, Siekkinen A, Xiong Y, Li Z, Xia Y. Facile Synthesis of Gold-Silver Nanocages with Controllable Pores on the Surface. J Am Chem Soc 2006; (128): 14776-14777.

39. Piao Y, Kim J, Na H, Kim D, Baek J, Ko M, Lee J, Shokouhimehr M, Hyeon T. Wrap-bake-peel process for nanostructural transformation from beta-FeOOH nanorods to biocompatible iron oxide nanocapsules. Nat Mater 2008; 7(3): 242-247.

40. Yang J, Lee J, Kang J, Lee K, Suh J, Yoon H, Huh Y, Haam S. Hollow Silica Nanocontainers as Drug Delivery Vehicles. Langmuir 2008; 24(7): 3417-3421.

41. Möller K, Bein T. Talented Mesoporous Silica Nanoparticles. Chem Mater 2017; 29(1): 371-388.

42. Waters L, Hussain T, Parkes G, Hanrahan J, Tobin J. Inclusion of fenofibrate in a series of mesoporous silicas using microwave irradiation. Eur J Pharm Biopharm 2013; 85(3): 936-941.

43. Zhu Y, Shi J, Shen $W$, Chen $H$, Dong $X$, Ruan $M$. Preparation of novel hollow mesoporous silica spheres and their sustained-release property. Nanotechnology 2005; (16): 2633-2638.

44. Vallet-Regí M. Revisiting ceramics for medical applications. Dalton Trans 2006; (44): 5211-5220.

45. Bouchoucha M, Gaudreault $R$, Fortin M, Kleitz $F$. Mesoporous Silica Nanoparticles: Selective Surface Functionalization for Optimal Relaxometric and Drug 
Loading Performances. Adv Funct Mater 2014; 24(37): 5911-5923.

46. She $X$, Chen L, Li C, He C, He L, Kong $L$. Functionalization of Hollow Mesoporous Silica Nanoparticles for Improved 5-FU Loading. J Nanomater 2015; (872035): 1-9.

47. Tang $Q, X u Y, W u D$, Sun $Y$. A study of carboxylicmodified mesoporous silica in controlled delivery for drug famotidine. J Solid State Chem 2006; 179(5): 1513-1520.

48. Popova M, Szegedi A, Kolev I, Mihály J, Tzankov B, Momekov G, Lambov N, Yoncheva K. Carboxylic modified spherical mesoporous silicas as drug delivery carriers. Int J Pharm 2012; 436(1-2): 778-785.

49. Wang $Y$, Sun $Y$, Wang J, Yang $Y$, Li Y, Yuan Y, Liu C. Charge-Reversal APTES-Modified Mesoporous Silica Nanoparticles with High Drug Loading and Release Controllability. ACS Appl Mater Interfaces 2016; (8): 17166-17175.

50. Shankland N, Wilson C, Florence A, Cox P. Refinement of Ibuprofen at $100 \mathrm{~K}$ by Single-Crystal Pulsed Neutron Diffraction. Acta Crystallogr Sect C 1997; (53): 951-954.

51. Song S, Hidajat K, Kawi S. Functionalized SBA-15 Materials as Carriers for Controlled Drug Delivery: Influence of Surface Properties on Matrix-Drug Interactions. Langmuir 2005; (21): 9568-9575.
52. Doadrio J, Sousa E, Izquierdo-Barba I, Doadrio A, PerezPariente J, Vallet-Regí $M$. Functionalization of mesoporous materials with long alkyl chains as a strategy for controlling drug delivery pattern. J Mater Chem 2006; 16(5): 462-466.

53. Moulari B, Pertuit $D$, Pellequer $Y$, Lamprecht $A$. The targeting of surface-modified silica nanoparticles to inflamed tissue in experimental colitis. Biomaterials 2008; (29): 4554-4560.

54. Yoncheva K, Popova M, Szegedi A, Mihály J, Tzankov B, Lambov N, Konstantinov S, Tzankova V, Pessina F, Valoti $M$. Functionalized mesoporous silica nanoparticles for oral delivery of budesonide. J Solid State Chem 2014; (211): 154-161.

55. Baranovskii V, Ganev V, Petkova V, Voicheva Kh, Dimitrov M. Hydrogels Based on Polycarboxylic AcidAgar-Agar Complexes. Colloid J 2012; 74(6): 675-679.

56. Voycheva Ch, Georgiev V, Natova M, Baranovsky V. Preparation, Modification and Characterisation of a Hydrogel Based on Polyacrylic and Polymetacrylic Acid as a Potential Carrier for Drug Delivery Systems. OAJPR 2017; 1(2): 000112.

57. Popova T, Voycheva Ch, Tzankov B. Study on the influence of technological factors on drug loading of poorly water-soluble drug on MCM-41 mesoporous carrier. Pharmacia 2020; 67(4): 351-356. 